\title{
Simulations of implicit learning of symmetries: The importance of prior knowledge and the nature of the memory buffer
}

\author{
Ruhai Zhang ${ }^{{ }^{\dagger}}$, Feifei $\mathrm{Li}^{2 \dagger}$, Shan Jiang ${ }^{13}$, Kexin Zhao ${ }^{1}$, \\ Chi Zhang ${ }^{1}$, Li Zheng ${ }^{1456^{*}}$, Lin Li ${ }^{16}$, Zoltán Dienes ${ }^{7}$
}

1. School of Psychology and Cognitive Science, East China Normal University, Shanghai, China.

2. Hangzhou College of Early Childhood Teacher Education, Zhejiang Normal University, Hangzhou, China

3. School of Government, Shanghai University of Political Science and Law, Shanghai, China

4. Shanghai Key Laboratory of Magnetic Resonance, School of Physics and Electronic Science, East China Normal University, Shanghai, China

5. Key Laboratory of Brain Functional Genomics, Ministry of Education, Shanghai Key Laboratory of Brain Functional Genomics, East China Normal University, Shanghai, China.

6. National Demonstration Center for Experimental Psychology Education, East China Normal University, Shanghai, China

7. School of Psychology, Sackler Centre for Consciousness Science, University of Sussex, Brighton, United Kingdom 
CA. Corresponding Author: Li Zheng

Li Zheng E-mail: lzheng@psy.ecnu.edu.cn

${ }^{\dagger}$ These authors contributed equally to this work. 


\begin{abstract}
The current research aimed to investigate the role that prior knowledge played in what structures could be implicitly learnt and also the nature of the memory buffer required for learning such structures. It is already established that people can implicitly learn to detect an inversion symmetry (i.e. a cross-serial dependency) based on linguistic tone types. The present study investigated the ability of the Simple Recurrent Network (SRN) to explain implicit learning of such recursive structures. We found that the SRN learnt the symmetry over tone types more effectively when given prior knowledge of the tone types (i.e. of the two categories tones were grouped into). The role of prior knowledge of the tone types in learning the inversion symmetry was tested on people: When an arbitrary classification of tones was used (i.e. in the absence of prior knowledge of categories), participants did not implicitly learn the inversion symmetry (unlike when they did have prior knowledge of the tone types). These results indicate the importance of prior knowledge in implicit learning of symmetrical structures. We further contrasted the learning of inversion symmetry and retrograde symmetry and showed that inversion was learnt more easily than retrograde by the SRN, matching our previous findings with people, thus showing that the type of memory buffer used in the SRN is suitable for modeling the implicit learning of symmetry in people.
\end{abstract}

Keywords: implicit learning, symmetry, inversion, retrograde, prior knowledge, Simple Recurrent Network 


\section{Introduction}

Implicit learning is an incidental learning process in which people become sensitive towards structures and regularities without needing to be aware of the knowledge acquired (Cleeremans, Destrebecqz, \& Boyer, 1998; Dienes, 2012; Janacsek \& Nemeth, 2015; Reber, Batterink, Thompson, \& Reuveni, 2019). Implicit learning has been argued to play a major role in different areas of human cognition, such as language acquisition (Paciorek \& Williams, 2015; Rebuschat, 2015; Rogers, Révész, \& Rebuschat, 2016; Saffran, Newport, Aslin, Tunick, \& Barrueco, 1997), social context (Lewicki, 1986; Norman \& Price, 2012), emotion (Jurchiş \& Opre, 2016), the perception of music (Rohrmeier \& Widdess, 2017; Tillmann, Bharucha, \& Bigand, 2000) and biological motion (Zhang, Li, Guo, Zheng, \& Zhou, 2020).

Key issues that the field of implicit learning has focused on are the content of the acquired knowledge and the type of mechanism or models that could account for learning such content. A simple theory is that people learn chunks of adjacent elements (Dulany, Carlson, \& Dewey, 1984; Perruchet \& Pacteau, 1990; Perruchet \& Vinter, 1998). Linguists have long argued against chunking as an explanation of language acquisition (e.g. Chomsky, 1959), suggesting rather that natural language could only be acquired by a mechanism that was able to deal with grammars above finite state (and even finite-state grammars can involve more than chunking) (e.g. Gazdar, Klein, Pullum, \& Sag, 1985; Joshi, Vijay-Shanker, \& Weir, 1991; Steedman, 2000). Relatedly, in the artificial grammar learning, researchers have argued, for example, that people can learn relations that are about non-local rather than just adjacent elements: either as long distance associations (Remillard, 2008); specific patterns found in learned exemplars (e.g. repetition patterns) (Brooks \& Vokey, 1991; Jamieson \& Mewhort, 
2009); or recursive rules (Dienes \& Longuet-Higgins, 2004; Jiang et al., 2012). One way of uniting the different accounts of what is learned is through a computational model such as the Simple Recurrent Network (SRN; Elman, 1990) that can learn a range of such structures (as shown by e.g., Christiansen, Dale, \& Reali, 2010; Onnis, Destrebecqz, Christiansen, Chater, \& Cleeremans, 2015; Rodriguez, Wiles, \& Elman, 1999) ${ }^{1}$. Leaning local associations or chunking involves minimal integration within or across stimuli; the way implicit learning can transcend chunking thus particularly sheds most light on the mechanisms by which it operates in storing and processing knowledge. Thus, using the SRN we will explore the principles by which knowledge is integrated within a stimulus and with past knowledge, using ecologically relevant structures that are more than finite state.

One structure that is above finite state is symmetry. Learning symmetry involves learning grammars above finite state, such as $\mathrm{A}^{\mathrm{n}} \mathrm{B}^{\mathrm{n}}$ (where a succession of $\mathrm{n}$ A elements is paired with a succession of n B elements) (for its learnability in the lab see e.g. de Vries, Monaghan, Knecht, \& Zwitserlood, 2008; Fitch \& Friederici, 2012; Lai \& Poletiek, 2013; Perruchet \& Rey, 2005; Poletiek, 2011; for the learnability of related recursive structures see Martins, Gingras, Puig-Waldmueller, \& Fitch, 2017; Martins, Martins, \& Fitch, 2016). For example, $\mathrm{A}^{1} \mathrm{~A}^{2} \mathrm{~A}^{3} \mathrm{~B}^{1} \mathrm{~B}^{2} \mathrm{~B}^{3}$, where the first $\mathrm{A}$ element requires the first $\mathrm{B}$ element be its corresponding partner, and likewise for the second and third (and any subsequent) elements, is a cross-serial dependency in language, or a mirror "inversion" in e.g. Serialist music (imagine putting a horizontal mirror under a music score). Several studies have shown that people can implicitly learn structures showing cross serial-dependencies (Dienes \& Longuet-Higgins, 2004; Kuhn

\footnotetext{
${ }^{1}$ The SRN must be modified to deal with e.g. backward associations (Mareschel \& French, 2017) or transfer across different terminals (Dienes, Altmann, \& Gao, 1999), but these issues do not bear on the structures considered in this paper.
} 
\& Dienes, 2005, 2006, with music; Uddén, Ingvar, Hagoort, \& Petersson, 2012, 2017, with letters; and Tanaka \& Watanabe, 2013, 2014, with visuomotor sequence learning; for evidence that people can implicitly learn recursive structure more generally see Rohrmeier, Fu, \& Dienes, 2012, and for a review, Rohrmeier, Dienes, Guo, \& Fu, 2014). Using musical inversion rules, studies found highly selected participants with an interest in Serialist music could implicitly learn to detect melodies instantiating the structure (Dienes \& Longuet-Higgins, 2004), while people who had no background could discriminate simple inversions from non-inversions on an indirect test (liking ratings, Kuhn \& Dienes, 2005) rather than on a direct test (grammaticality judgment, Kuhn \& Dienes, 2005, 2006).These studies demonstrated the acquisition of unconscious knowledge of inversion, suggesting that implicit learning of inversion was affected by prior knowledge people might have.

Consistently, there is evidence that implicit learning is sensitive to prior knowledge in other contexts (e.g. Chen et al., 2011, Leung \& Williams, 2011, for implicit learning of form-meaning connections; see also Ziori \& Dienes, 2006, 2008, for implicit concept formation; and Ziori, Pothos, \& Dienes, 2014 for artificial grammar learning). Therefore, an issue raised is in what way prior knowledge constrains how inversions/cross-serial dependencies are learned in a given situation.

We will explore a possible computational mechanism for learning inversions by reference to a paradigm introduced by Jiang et al. (2012) (and explored further by Li, Jiang, Guo, Yang, \& Dienes, 2013, Ling, Li, Qiao, Guo, \& Dienes, 2016, and Qiao et al., 2018). By controlling both chunks and repetition structures, Jiang et al. showed that Chinese participants could implicitly learn to discriminate lexical tonal inversions from non-inversions. Chinese is 
a tonal language that uses four tones to signal different meanings; for example, the syllable "ma" pronounced in tone 1 could mean "mother", but "horse" when in tone 3 . Tone 1 , tone 2 , tone 3 , and tone 4 indicate flat, rising, falling-rising and falling phonetic characteristics in pitch respectively. Tone 1 and tone 2 are categorized into ping (level) tones, while tone 3 and tone 4 are categorized into ze (oblique) tones in Chinese. The grammar used in Jiang et al.'s study was an inversion rule of the two tone types, where tones in successive lines bear an inversion relation to each other. Specifically, if a tone for a syllable in a certain position in the first line is one category (e.g. ping), the syllable in the same position in the second line would be in the other category (e.g. ze), and vice versa (see Fig.1). The way of categorizing four tones into ping or ze tones is taught to Chinese participants at school. Thus, do the participants implicitly learn about inversion of ping-ze tones because ping-ze categories constitute prior knowledge for them? This is an issue which will be addressed by using simulation as well as a human experiment.

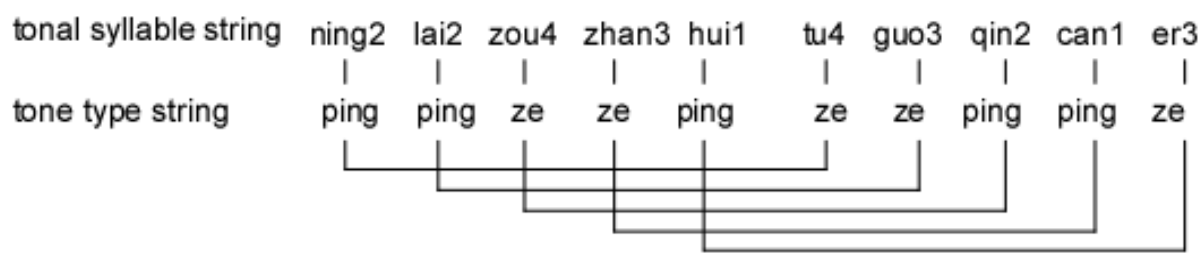

Fig. 1. An example of grammatical strings of the inversion rule in Jiang et al. (2012) study. Each string consisted of 10 tonal syllables and the tone types (pings or zes) of the previous five syllables predict the following inversion, e.g., if the tone type of the first syllable was ping, then the tone type of the sixth syllable was ze, and if the tone type of the second syllable was ping, then the tone of the seventh syllable was ze, and so on. The inversion relation can 
be construed as an element to element mapping as shown.

Generally, learning of non-local dependencies like an inversion generated by above finite-state grammars requires a memory buffer, as the Simple Recurrent Network (SRN) uses (see Fig. 2). The SRN has a context layer, which provides a temporary storage buffer, in principle enabling the SRN to learn grammars more powerful than finite-state grammars (e.g. Christiansen \& Chater, 1999). Indeed, studies have showed that the SRN can learn structures instantiating inversions, at least in term of the long-range dependencies that inversions produce (Kuhn \& Dienes, 2008). The dependence of how an SRN learning inversions depends on prior knowledge has yet to be evaluated (cf. Altmann, 2002, for the importance of prior experience for SRN).

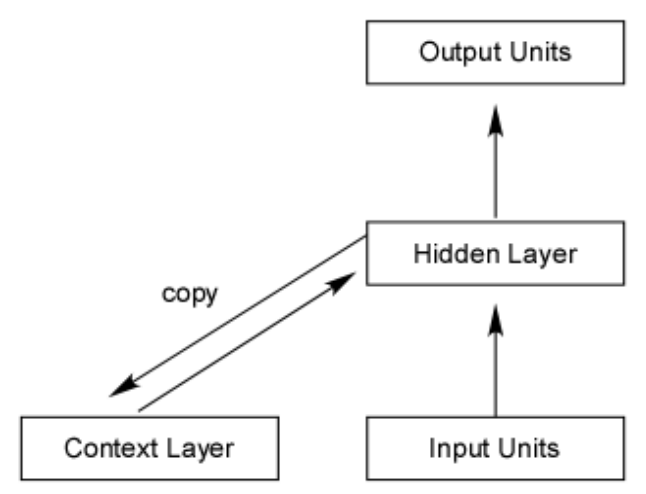

Fig. 2. Schematic diagram of the SRN (Kuhn \& Dienes, 2008). The SRN is a three-layered feed-forward network consisting of an extra set of units (context layer) which is a copy of the hidden layer from the previous time step that then feed back into the hidden layer; thus, at time $t$ the activation of the hidden units is influenced by both the input activation and the activation of the hidden units at time $t-1$. 
Specifically, in the study of Jiang et al. (2012), the inversion was defined over ping-ze classes of tones rather than four tones. Therefore, by contrasting different ways the materials were coded for the SRN, i.e. coding over ping-ze classes or four tones, we could investigate the role of prior knowledge of tone types played in implicit learning of tonal inversion. If knowledge of ping-ze categories facilitates implicit learning, and the SRN is a good model of implicit learning, then the SRN should show better performance when a ping-ze coding rather than a tone coding is used; if knowledge of ping-ze categories doesn't influence implicit learning, there should be no difference in the performance of the SRN between the two ways of coding. We presume the tones themselves can be perfectly well perceived, a form of prior knowledge that is held constant (but not culturally universal, Caldwell-Harris, Ladd, Dediu, Biller, \& Christiansen, 2015); it was prior knowledge in the superordinate category of the tone that was varied (i.e., which two tones belong to the ping category and which to the ze category). The first aim of the present research was to investigate the role of prior knowledge by using two ways of coding the input to the SRN for the materials of Jiang et al. (i.e. ping and ze categories given to the SRN, or else the two categories have to be induced from the four tones).

The second aim of the current research was to investigate further the effect of prior knowledge of tone types on implicit knowledge of tonal inversion by a human experiment. If the participants implicitly learn about inversion of ping-ze tones in the study of Jiang et al. (2012) because ping-ze categories constituted prior knowledge for them, what would happen if we used an arbitrary classification of four tones into two categories? The second aim of the 
current research was to investigate this issue by using an arbitrary classification of four tones, e.g., categorizing tone 1 and tone 3 as "ping" tones, and categorizing tone 2 and tone 4 as "ze" tones (see Fig. 3). The inability to classify would be evidence that people do not readily learn to detect inversion symmetry when they also need to induce the categories, a result that would help argue for the effect of prior knowledge on implicit learning.

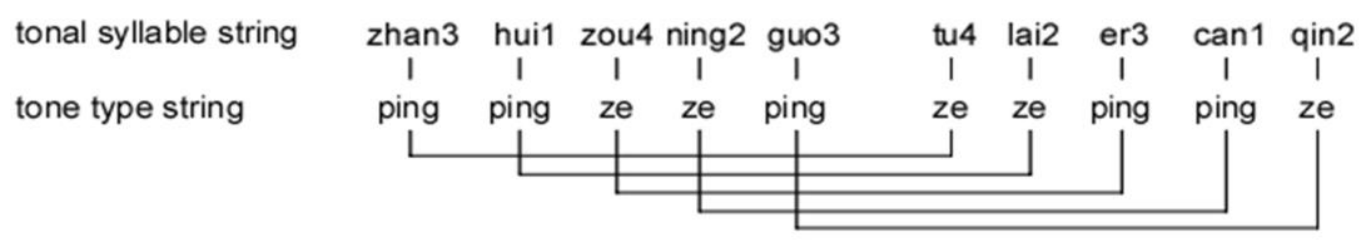

Fig. 3. An example of grammatical strings of the inversion rule in which tone $1 \&$ tone 3 were redefined as the new ping tones and tone $2 \&$ tone 4 were redefined as the new ze tones.

In addition, if the SRN can learn tonal inversion with either type of coding, it would illustrate that implicit learning of symmetry requires a memory buffer (Kuhn \& Dienes, 2008; Misyak, Christiansen, \& Tomblin, 2009; Servan-Schreiber, Cleeremans, \& McClelland, 1991). However, it remains an open issue as to what the nature of the buffer is, an issue which we will address by using symmetries. Besides inversion, another type of symmetry is a mirror "retrograde" in music, e.g. a melody CEB-BEC (Balch, 1981; think of the music score for the first half being reflected in a vertical mirror to obtain the second half). Dienes and Longuet-Higgins (2004) discussed how, by contrasting the implicit learning of inversion and retrograde, researchers could investigate the nature of the memory buffer required for processing structure beyond finite state. A first in-first out buffer, which outputs material in 
the same order it was inputted, will facilitate detecting inversion, whereas a last in-first out buffer, which outputs material in the reverse order to which it was inputted, will facilitate detecting retrograde (Christiansen \& Chater, 1999; Dienes \& Longuet-Higgins, 2004; Li et al., 2013; Uddén et al., 2012). Thus, investigating implicit learning of inversion and retrograde is theoretically valuable for modeling in terms of constraining the computational properties of the memory buffer involved in the implicit learning of sequences.

Uddén et al. (2012) demonstrated that participants performed better on materials instantiating inversions than retrogrades in an artificial grammar learning paradigm involving strings of letters (with a mapping between two pairs of letters, F-L, D-P). Nonetheless, the repetition structures were not counterbalanced between inversions and retrogrades. Thus, participants may have simply memorized repetition patterns, a strategy for which there is evidence in artificial grammar learning (e.g. Tunney \& Altmann, 2001). Participants did not report the symmetry rules in post task report; but maybe they did not learn symmetries, implicitly or explicitly. By controlling both repetition structures and chunks, Li et al. (2013) contrasted the implicit learning of Chinese tonal inversions and retrogrades on human participants. The materials were constructed so that the tone types in successive lines formed an inversion or a retrograde to each other (see Fig. 4). Results demonstrated that people acquired unconscious structural knowledge of both inversions and retrogrades. Moreover, inversions were implicitly learnt more easily than retrogrades, suggesting that functionally the memory buffer used in implicit learning is more like a first in-first out buffer than a last in-first out buffer. Therefore, the third aim of the current research was to examine whether the SRN could replicate the advantage of inversions over retrogrades, in order to explore whether 
it models the functional properties required of the memory buffer in implicit learning.

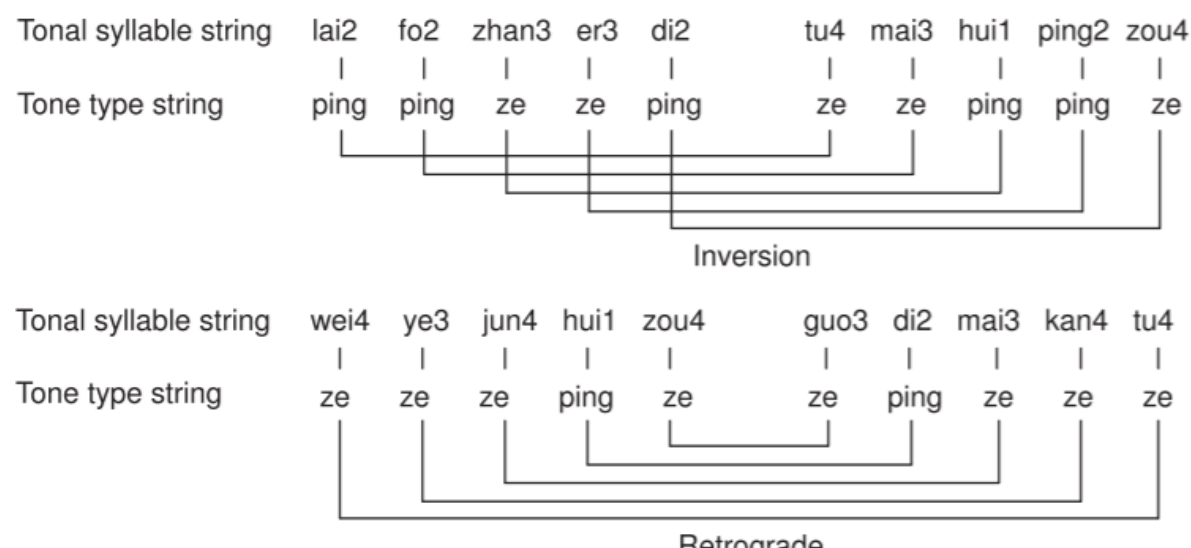

Fig. 4. Examples of grammatical strings of the retrograde and inversion (Li et al., 2013). The tone types (pings or zes) of the previous five tonal syllables predict the following five tonal syllables by forming a retrograde or an inversion.

In summary, this research investigated the role prior knowledge played in what structures could be implicitly learnt and the nature of memory buffer required for learning such structures. Study 1 was to make predictions concerning the effect of prior knowledge of tone types on implicit learning of tonal inversion by using SRN simulations. Specifically, we trained and tested the SRN on the same materials used by Jiang et al. (2012). Two ways in which the materials were coded were used, i.e. coding over ping-ze classes or four tones. Study 2 sought to the prediction coming from the simulations (if clear predictions were made) on people by using an arbitrary classification of four tones, i.e. categorizing tone 1 and tone 3 into ping tones and categorizing tone 2 and tone 4 into ze tones, or categorizing tone 1 and tone 4 into ping tones and categorizing tone 2 and tone 3 into ze tones (see Fig. 3). In training, participants listened to and then silently repeated the strings. In a subsequent test, participants 
classified new syllable strings as grammatical or not. Because we are interested in the nature of specifically implicit learning, a methodology is needed to determine the implicit nature of the knowledge. In the test phase, after the classification judgment, people then gave the structural knowledge attributions of Dienes and Scott (2005) to assess awareness on a trial by trial basis. Study 3 aimed to examine the nature of the memory buffer required for learning symmetrical structures beyond finite state. We trained and tested the SRN on the same materials used by Li et al. (2013), and established which of inversions or retrogrades were easier, in order to explore the functional properties required of the memory buffer in implicit learning ${ }^{2}$. Together, the studies explore the way how knowledge is integrated within a string (the long range dependencies present in inversion vs. retrogrades) and across contexts (the role of prior knowledge) in implicit learning, and the capacity of the SRN to model not only existing data (inversions vs. retrogrades) but also to make predictions (about the role of prior knowledge).

\section{Study 1}

The aim of study 1 was to investigate the effect of prior knowledge of tone types on the implicit learning of tonal inversion by using SRN simulations. Specifically, we trained and tested the SRN on the same materials used by Jiang et al. (2012). Two ways in which the

\footnotetext{
${ }^{2}$ For Study 3, by using the same simulation code and materials, data were obtained and published in a study of Li \& Liu (2018) in a Chinese journal (Journal of Psychological Science, ISSN 1671-6981; Paper title: Which memory buffer does the implicit learning mechanism of nonlocal dependencies use: Evidence from neural network simulations, DOI:

10.16719/j.cnki.1671-6981.20180405). Though there is an abstract written in English, more important and detailed information could be found only in the body of the article which is written in Chinese, limiting readability of the article for people who do not understand Chinese. Considering that the SRN simulation in Study 3 is of value for understanding the nature of the memory buffer used in implicit learning, we include Study 3 in the current paper. Additionally, though similar conclusions were made in the two papers, the data are different because fewer training epochs (but bringing about similar discrimination performance of SRNs and human participants; $10 \mathrm{vs} 100$ epochs) were used in the current paper compared to the Chinese paper in order to provide novel data, and further, training conditions more similar between models and humans.
} 
materials were coded, i.e. coding over ping-ze classes or over the four tones. If knowledge of ping-ze categories facilitates learning in the SRN, the SRN, as a model of implicit learning, makes the corresponding prediction for people. Conversely, if knowledge of ping-ze categories does not appreciably influence learning in the SRN, there should be little difference in the performance of people who have prior knowledge of the categories and those that do not.

\subsection{Method}

\subsubsection{Materials}

The SRN were tested on the same training and test materials of Chinese tones of Jiang et al. (2012). In this study, the grammar was an inversion rule of two tone types (pings and zes), where the inversion of ping was ze, and vice versa. The ping tones contained tone 1 and tone 2, while the ze tones contained tone 3 and tone 4 . A total of 12 tonal syllables were selected, six syllables of which were ping tones and the other six syllables of which were ze tones. Each string consisted of 10 different tonal syllables. Grammatical strings were created according the inversion rule that the tone types of previous five syllables predicted the following five syllables (see Fig. 1, cf. Jiang et al., 2012), whereas ungrammatical strings were created by exchanging the last one, two or three tone types of the previous five elements for the first one, two or three ones of the following five elements of the grammatical strings, ensuring two elements violated the inversion rule. Finally, 48 grammatical strings with different syllables were created and then were repeated three times for constructing 144 syllable strings in all which were as served as training strings. The 64 new syllable strings, including 32 grammatical syllable strings and 32 ungrammatical syllable strings, were created 
and used as test strings (cf. Jiang et al., 2012, which uses the same stimuli).

The materials controlled both repetition structures and chunks. None of the test strings had the same repetition structures as any of the training strings in terms of tone types (ping or ze) or tones 1-4. And the repetition structures of syllables were the same for all training and test strings. With respect to chunks, mean feature frequency (MFF), global anchor associative chunk strength (GACS) and anchor associative chunk strength (AACS) were matched between grammatical and ungrammatical test strings in terms of tone types, tones 1-4 and tonal syllables (cf. Jiang et al., 2012).

\subsubsection{Procedure}

The networks were assigned to four cells of the two training conditions (trained vs. untrained) by two coding conditions (ping-ze coding vs. tone coding) design to simulate experiments on human participants. As in Jiang et al.'s experiment (2012), the networks' learning was evaluated by comparing the trained networks' performance with that of untrained networks (see also Kuhn \& Dienes, 2008). Only the trained groups received the training phase, while all the four groups received the test phase.

Training phase. The SRN had the same number of input units as output units and one hidden layer. During training, strings were presented one element at a time by activating the input units, and the network was trained to predict the next element in the sequence. The error between the target and the output activation was used to update the weights by using backpropagation. In ping-ze coding condition, two input/output units represented the ping and ze tones respectively. While in tones coding condition, four input/output units represented tone 1 , tone 2 , tone 3 and tone 4 . The activation of the appropriate unit was then set to 0.9 and 
all other units were set to 0.1 . A complete string could be represented as a vector by concatenating the vectors specifying the unit activations for each successive element. For each network, the 144 training strings were presented in a different random order.

Test phase. In the test phase, networks were presented with the 64 test strings, and their ability to predict the next element in the sequence was used as an index of performance. If the network has acquired knowledge about the training strings it would be expected to perform better on the grammatical than on the ungrammatical strings. Performance was assessed by calculating the cosine (COS) of the angle between the target vector $t$ and the output vector $o$ (e.g. Altmann \& Dienes, 1999; Dienes et al., 1999; Kinder, 2000; Kuhn \& Dienes, 2008). The larger the COS, the better the performance is. In order to compare the networks' discrimination performance to that of the human participants, $\mathrm{z}$-scores were calculated by subtracting the ungrammatical strings' mean COS from the grammatical string' mean COS, and dividing this difference by the pooled standard deviation. A positive score represents an ability to discriminate between grammatical and ungrammatical strings, and 0 is chance performance. Considering that only the second five elements could be predicted by the first five elements according to the two rules, we calculated the z-scores just for the predictable second five elements. During the test phase, training was left on; presumably, learning devices in the brain do not switch themselves off in test phases.

The code, materials and raw data are available here:

https://osf.io/6arzx/?view_only=222c7ba6289c487996860bcf093b17c7

\subsubsection{Networks parameters}

The networks were trained and tested using a large set of parameter values. This 
approach led to a parameter space, rather than a single best fit value. Determining the characteristic behavior of the model and then evaluating the models in terms of all of these parameters allows the model to have explanatory power (Boucher \& Dienes, 2003; Kuhn \& Dienes, 2008).

For each condition, the simulations were carried out using all possible permutations of the parameter values presented in Table 1. The selection of these parameters was based on existing artificial grammar learning simulations (Cleeremans \& Dienes, 2008). All of the parameters combinations resulted in 150 different models. Each of these models was run 25 times, each time using a different set of random starting weights, using the Nguyen-Widrow method (1990). All networks were simulated using the Matlab Neural Network toolbox.

\section{Table 1}

Range of parameter values used in the simulations.

\begin{tabular}{ll}
\hline Network parameter & Value \\
\hline Learning rate & $0.1,0.3,0.5,0.7,0.9$ \\
Momentum & $0.1,0.3,0.5,0.7,0.9$ \\
Number of hidden units & $5,10,15,30,60,120$ \\
Epochs & 10 \\
\hline
\end{tabular}

\subsection{Results and discussion}

\subsubsection{Performance of networks}

Table 2 shows the mean z-scores for each group of the networks. Confidence intervals (or credibility intervals with a uniform prior) showed that trained networks performed substantially better than the untrained networks for ping-ze coding, 95\% CI [0.37, 0.55], and 
trained networks could only outperform untrained networks to a small degree for tone coding, $95 \%$ CI $[0.02,0.04]$. That is, the SRN successfully discriminated between grammatical and ungrammatical strings in the absence of chunking cues, especially for ping-ze coding.

Untrained networks in ping-ze coding condition did not perform substantially different from the untrained networks in tone coding condition 95\% CI [-0.006, 0.02]. Crucially, trained networks in ping-ze coding condition performed substantially better than trained networks in the tones coding condition, $95 \%$ CI $[0.35,0.53]$. The substantially better performance of the SRN when a ping-ze coding rather than a tone coding was used indicated that the knowledge of ping-ze categories facilitated the learning of tonal inversion.

("Substantially" different as used here can be scaled by reference to human data; see below.)

\section{Table 2}

Mean $\mathrm{z}$-scores for trained and untrained networks in two coding conditions.

\begin{tabular}{ccccccc}
\hline & \multicolumn{2}{c}{ Trained } & & \multicolumn{2}{c}{ Untrained } \\
\cline { 2 - 3 } \cline { 6 - 7 } Coding condition & $\mathrm{M}$ & $\mathrm{SE}$ & & $\mathrm{M}$ & $\mathrm{SE}$ \\
\hline Ping-ze coding & 0.47 & 0.05 & & 0.01 & 0.00 \\
Tone coding & 0.03 & 0.00 & & 0.00 & 0.00 \\
\hline
\end{tabular}

\subsubsection{Characteristic behavior of the networks}

We evaluated how typical the performance of the networks was to that compared to the human data. The effects of learning were calculated for each network by subtracting the z-scores of the untrained networks from the z-scores of the trained networks. Similarly, human participants' learning was assessed by subtracting the mean z-scores of the control 
group from that of the experimental group. The differences in z-scores in the ping-ze coding and tone coding conditions were then plotted (see Fig. 5). In Jiang et al.'s experiment (2012), the discrimination performance of the human participants $\left(d^{\prime}\right)$ for experimental and control groups were $0.40(S E=0.08)$ and $0.06(S E=0.05)$, respectively. The difference between the experimental and control groups (mean $\pm 1 S E$ ) of human's performance was $0.25-0.44$. The area covered by the human participants is also shown in Fig. 5.

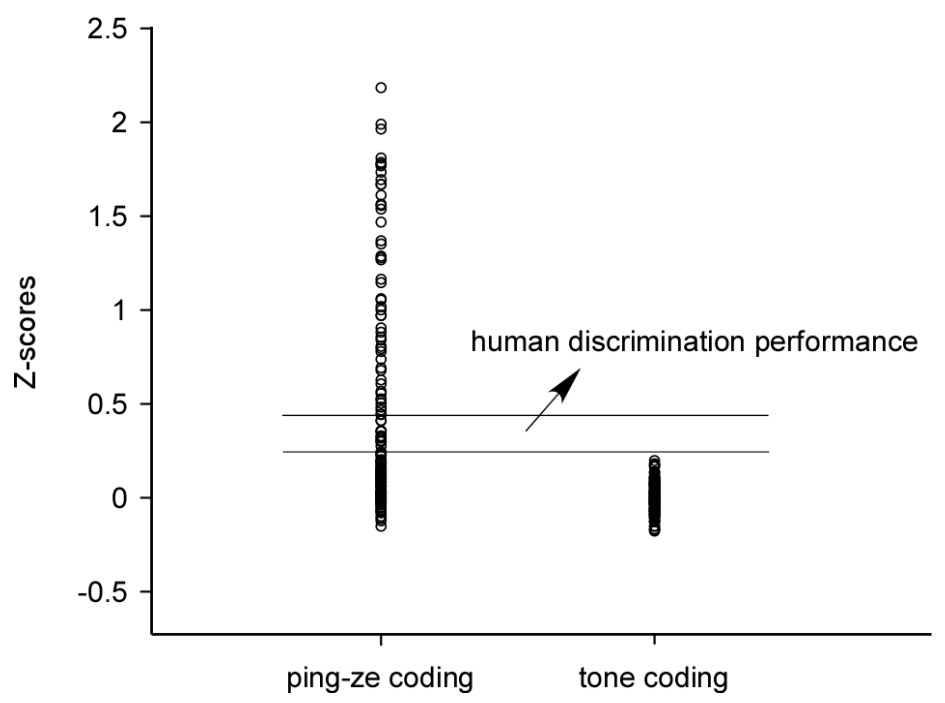

Fig. 5. Scatter plot shows the difference between the trained and untrained networks' z-scores in the ping-ze coding condition and the z-scores in the tone coding condition. The plot also shows the areas covered by the human discrimination performance (mean $\pm 1 S E$ ).

With regards to the human performance, the characteristic discrimination performance of models with ping-ze coding appeared to somewhat match the human data, as shown by the fact that a substantial number of the SRNs fell within the area covered by the human data (16/150 in ping-ze coding condition), while there was no SRNs with tone coding condition) fell within the area covered by the human data, suggesting that the characteristic performance 
of the SRN matches reasonably well people's learning of tonal inversion with prior knowledge of ping-ze categories.

\section{Study 2}

Study 1 showed the SRN makes the clear prediction that learning the inversion rule without prior knowledge (i.e., how to divide the four terminals into the two categories over which the inversion is defined) should be very difficult. We test this prediction on human participants.

\subsection{Method}

\subsubsection{Participants}

Twenty-six volunteers (15 female, aged $18-26, M=22.54, S D=1.96)$ from the university community participated in return for money. None of the participants reported a history of hearing difficulties. All the participants were native Chinese speakers and gave written informed consent before the experiment. The study was approved by the Ethics Committee on Human Experiments of East China Normal University.

\subsubsection{Design}

This experiment Grammaticality (grammatical vs. ungrammatical) was the within-participants factor. The dependent variable was the proportion of test strings classified correctly.

\subsubsection{Materials}

The experiment used the same materials as Jiang et al. (2012), with a change in how the 
four tones mapped onto the two categories over which the inversion rule was defined. In the study of Jiang et al. (2012), a total of 12 tonal syllables were used: "cān jū huī (tones 1) níng lái qín (tones 2) guǒ ěr zhăn (tones 3) zòu tù jùn (tones 4)” (to help readers understand, these labels were used: can1 jü1 hui1 ning2 lai2 qin2 guo3 er3 zhan3 zou4 tu4 jün4), where tones 1 \& tones 2 were categorized as ping tones and tones $3 \&$ tones 4 were categorized as ze tones in the traditional way. In this study, the new ping tones and the new ze tones were created and used to build the strings according to the inversion rule, where the inversion of new ping tone was new ze tone, and vice versa (see Fig. 3). For half of the participants, tones $1 \&$ tones 3 were redefined as the new ping tones and tones $2 \&$ tones 4 were the new ze tones, while for the other half, tones $1 \&$ tones 4 were redefined as the new ping tones and tones $2 \&$ tones 3 were the new ze tones. By redefining ping and ze tones, the 48 new grammatical training strings and 64 new test strings (32 grammatical strings and 32 ungrammatical strings) with different syllables were conducted on the basis of materials of Jiang et al. (2012), with matched repetition structures between training and test strings and matched chunks (MFF, GACS and AACS) between grammatical and ungrammatical test strings in terms of tone types, tones 1-4 and tonal syllables (cf. Jiang et al., 2012).

Materials and raw data of the experiment are available here: https://osf.io/6arzx/?view_only=222c7ba6289c487996860bcf093b17c7

\subsubsection{Procedure}

The experiment was performed with the same procedure of Jiang et al. (2012). Specifically, there were two phases: the training and the test phase. In the training phase, 48 grammatical strings were repeated three times in a random order. In each trial, a warning tone 
was presented for $450 \mathrm{~ms}$, followed by a $5100 \mathrm{~ms}$ syllable string (10 tonal syllables, each lasting for $450 \mathrm{~ms}$, with a $600 \mathrm{~ms}$ interval between the fifth and sixth syllables to create a perceptual gap between the first half of the string and its inversion in the final half) and a $5000 \mathrm{~ms}$ blank. Participants were instructed to listen to each string carefully and silently repeat it during the $5000 \mathrm{~ms}$ delay before the next trial.

During test phase, participants were informed that the strings that they heard in the training phase were generated using a specific rule and 64 new strings would be presented in a random order. For each string, they were required to judge whether the given string was grammatical or not and then choose their judgment basis from four categories (guess, intuition, memory and rules). As defined to participants, "guess" indicated that the judgment was based on nothing at all, it could just as well be based on a toss of a coin; "intuition" indicated that the judgment was based on a hunch or feeling that could not be explicated further, i.e., there was confidence in the judgment but the person had no idea why the judgment was right; "memory" indicated that the judgment was based on a recollection; "rules" indicated the judgment was based on a rule that could be stated if asked (for previous uses of these structural knowledge attributions see e.g. Dienes \& Scott, 2005; Jiang et al., 2012; Jurchiș, Costea, Dienes, Jurchiş \& Opre, 2016; Miclea \& Opre, in press; Neil \& Higham, 2012; Norman \& Price, 2012; Qiao et al, 2018; Waroquier, Abadie, \& Dienes, in press). 'Guess' and 'intuition' responses can be combined together as potential cases of unconscious structural knowledge, and 'memory' and 'rules' responses combined together as potential cases of conscious structural knowledge (Dienes, 2012).

\subsection{Results and discussion}


The proportion of correct response was calculated by $\frac{N c+0.5}{N+1},(\mathrm{Nc}$ being the number of correct responses; and $\mathrm{N}$ the total number of responses), the correction corresponding to a Bayesian prior of chance performance worth just one observation, useful when data are limited (similar to Laplace's correction to a proportion, but using a "unit information prior," i.e. a prior worth one observation; cf. Baguley, 2012, pp. 83 and 397). One outlier in the proportion of correct response was identified by use of a Stem and Leaf graph and excluded from further statistical analyses. Jiang et al. (2012) using the same materials but with the classic ping-ze categories obtained an overall performance of $58 \%$ correct. Thus, if participants could learn the current materials in similar was those of Jiang et al, we can model H1 with an expected scale of effect of 0.08 above baseline (the chance level 0.5 ); specifically we used a half-normal with an SD of 0.08 (Dienes, 2014). We assessed evidence for this H1 versus $\mathrm{H} 0$ by a Bayes factor, $B_{\mathrm{H}(0,0.08)}$, where the notation indicates a half-normal (' $\mathrm{H}$ '), with a mode of 0 and an SD of 0.08. Following Jeffreys (1939/1961), we will regard a $B$ above about 3 as substantial evidence for $\mathrm{H} 1$ versus $\mathrm{H} 0$; thus, a $B$ below a $1 / 3$ is substantial evidence for $\mathrm{H} 0$ over H1. To indicate the robustness of Bayesian conclusions, for each $B$, a Robustness Region is reported, giving the range of scales that qualitatively support the same conclusion (i.e. evidence as supporting $\mathrm{H} 0$, or as supporting H1, or there not being much evidence at all), notated as: $\mathrm{RR}_{\mathrm{B}<1 / 3[\mathrm{x} 1, \mathrm{x} 2]}$ where $\mathrm{x} 1$ is the smallest $\mathrm{SD}$ that gives the same conclusion and $\mathrm{x} 2$ is

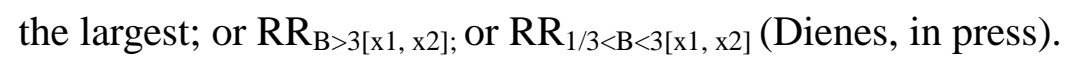

The classification performance in the test phase was $0.50(S D=0.06)$, when compared to the chance level $0.50, t(24)=-0.20, p=.84,95 \%$ CI $[-0.03,0.02]$, Cohen's $d=0.04, B_{\mathrm{H}(0,0.08)}$ $=0.13, \mathrm{RR}_{B}<1 / 3[0.03,>0.5]$, showing evidence for the null hypothesis that people could not 
learn the inversion when having to induce new categories, as predicted by the SRN modeling. We also compared data of Jiang et al. (2012) and the present study, $t(44)=3.97, p<.001$, $95 \%$ CI $[0.04,0.12]$, Cohen's $d=1.18, B_{\mathrm{H}(0,0.08)}=253.74, \mathrm{RR}_{B}>_{3}[0.01,>0.5]$, providing further evidence for the influence of prior knowledge of ping-ze categories on learning tonal inversions.

Additionally, 'guess' and 'intuition' responses were combined together as indicators of unconscious structural knowledge (implicit attributions), and 'memory' and 'rules' responses were combined together as indicators of conscious structural knowledge (explicit attributions). Implicit attributions were given a large proportion of the time $(84 \%, 95 \%$ CI [74\%, 93\%]). By use of a Stem and Leaf graph, three outliers in the response proportion were identified. We found that mean proportion of explicit attributions was only $9 \%(S D=8 \%)$ with the outliers removed (the response accuracy of explicit attributions of 22 participants was $0.51 \pm 0.15$, 95\% CI $[0.44,0.57])$, indicating participants' grammaticality judgments were mainly based on implicit attributions. Thus we focused on implicit attributions in the following analyses. It was found that the response accuracy of implicit attributions was $0.50(S D=0.07)$. When compared to the chance level $0.50, t(21)=-0.17, p=.87,95 \%$ CI [-0.03, 0.03], Cohen's $d=$ $0.04, B_{\mathrm{H}(0,0.06)}=0.22, \mathrm{RR}_{B}<1 / 3[0.04,>0.5]$ (Given that Jiang et al. (2012) obtained the performance of $56 \%$ correct for implicit attributions, here the Bayes factor was calculated with the expected scale of effect of 0.06 above baseline). We further compared data of Jiang et al. (2012) and the current study, $t(41)=2.61, p=.012,95 \%$ CI [0.01, 0.11], Cohen's $d=0.80$, $B_{\mathrm{H}(0,0.06)}=11.57, \mathrm{RR}_{B}>{ }_{3}[0.02,0.33]$, suggesting that prior knowledge of ping-ze categories played an important role in learning tonal inversions implicitly. 


\section{Study 3}

Study 3 aimed to examine the nature of the memory buffer required for learning symmetrical structures beyond finite state. We trained and tested the SRN on the same materials used by Li et al. (2013), and established whether retrogrades or inversions were easier, in order to explore whether the functional properties required of the memory buffer in implicit learning corresponded to those of the SRN.

\subsection{Method}

\subsubsection{Materials}

The SRN was tested on the same training and test materials of Li et al. (2013). In their study, both an inversion and a retrograde rule of two tone types (pings and zes, defined classically, i.e. not as we did in Study 2) were used. For each rule, 144 grammatical strings were studied in the training phase and 48 new strings ( 24 grammatical strings and 24 ungrammatical strings) were tested in the test phase. Each string consisted of 10 different tonal syllables, where the tone types of the first five syllables predicted the tone types of the following five by forming an inversion or a retrograde in the grammatical strings (see Fig. 3). Ungrammatical strings in the test phase violated non-local dependency in the seventh and ninth positions (for the retrograde rule, dependencies were violated in second-ninth and fourth-seven pairs; for inversion rule, dependencies were violated in second-seven and fourth-ninth pairs). For each rule, repetition structures were matched between training and test strings, and chunks (MFF, GACS and AACS) were matched between grammatical and ungrammatical strings in terms of tone types, tones 1-4 and tonal syllables (cf. Li et al., 2013). 


\subsubsection{Procedure}

The networks were assigned to four cells of the two training conditions (trained vs. untrained) by two rules (inversion vs. retrograde) design to simulate experiments on human participants. Only the trained inversion group and trained retrograde group received the training phase, while all the four groups received the test phase.

Training phase. The same 144 strings as human participants were used for two groups of trained networks. Strings were presented one element at a time by activating the input units, and the networks were trained to predict the next element in the sequence. The error between the target and the output activation was used to update the weights by using backpropagation. Two input/output units represented the two tone types, considering that ping-ze is an existing category for subjects and such a category is needed for the learning to occur in humans (see Study 1 and 2). The activation of the appropriate unit was set to 0.9 and the other unit was set to 0.1 . For each network, the sequence was presented in a different random order.

Test phase. Networks were presented with 48 test sequences, and their ability to predict the next tone in the sequence was used as an index of performance. Performance was assessed by calculating the z-scores for the predictable second five elements as Study 1. And during the test phase, training was left on as Study 1.

For each condition, the simulations were carried out using the same 150 parameters combinations as Study 1 (see Table 1). Each of these models was run 25 times, each time using a different set of random starting weights, using the Nguyen-Widrow method (1990). All networks were simulated using the Matlab Neural Network toolbox.

The code, materials and raw data are available here: 
https://osf.io/6arzx/?view_only=222c7ba6289c487996860bcf093b17c7

\subsection{Results and discussion}

\subsubsection{Performance of networks}

Table 3 shows the mean z-scores for each group of the networks. Credibility intervals (with uniform priors) showed that trained networks performed substantially better than untrained networks for both inversion, $95 \%$ CI $[0.49,0.74]$ and retrograde groups, $95 \%$ CI $[0.16,0.25]$. Moreover, for trained groups, the inversion group performed substantially better than the retrograde group, $95 \%$ CI $[0.32,0.51]$. For untrained groups, the inversion group performed similarly poorly as the retrograde group, $95 \%$ CI $[-0.02,0.02]$.

The fact that the SRN successfully discriminated between grammatical and ungrammatical sequences in the absence of chunking cues shows that the networks learnt to detect the inversion and retrograde structures, as instantiated in our materials. In addition, the substantial performance difference between inversion and retrograde for trained rather than untrained networks indicates that inversions were learnt more easily than retrogrades, which coincides with the result of human experiment (Li et al., 2013).

\section{Table 3}

Mean z-scores for trained and untrained networks and the discrimination performance of the human participants (Li et al., 2013) for two rules.

\begin{tabular}{cccccccccc}
\hline & \multicolumn{4}{c}{ SRN } & \multicolumn{5}{c}{ Human } \\
\cline { 2 - 10 } Rule & \multicolumn{2}{c}{ Trained } & \multicolumn{2}{c}{ Untrained } & \multicolumn{2}{c}{ Experimental } & \multicolumn{2}{c}{ Control } \\
\cline { 2 - 10 } & $\mathrm{M}$ & $\mathrm{SE}$ & $\mathrm{M}$ & $\mathrm{SE}$ & $\mathrm{M}$ & $\mathrm{SE}$ & $\mathrm{M}$ & $\mathrm{SE}$ \\
\hline Inversion & 0.63 & 0.06 & 0.01 & 0.01 & 0.47 & 0.16 & -0.02 & 0.07 \\
Retrograde & 0.21 & 0.02 & 0.01 & 0.01 & 0.28 & 0.17 & -0.07 & 0.05 \\
\hline
\end{tabular}




\subsubsection{Non-local dependency learning by $S R N$}

Discrimination performance was averaged across the two rules, and the parameter values of the network with the highest overall performance were used for the subsequent analyses (cf. Kuhn \& Dienes, 2008). The SRN containing 60 hidden units, a learning rate of 0.7 , and a momentum of 0.5 led to the best overall discrimination performance. So we chose the data of the 25 simulations of this network for further analyses. If the SRN has learnt the non-local mapping, then once the network has been presented with a particular input element, it should be able to correctly predict the associated item in the correct position. For example, if the network is presented with a ping tone in the first position, it should be able to predict a ze tone in the sixth position according to inversion rule, as this was one of the mappings the network was trained to learn, regardless of what the intervening material is. Thus, a direct way to gain an insight into what the network has actually learnt is investigating the performance of the trained networks in each predictable position of grammatical and ungrammatical sequences. Given that the ungrammatical sequences were created with violations in the seventh and ninth positions for both rules, it could be predicted that the performance of only the trained networks in these two positions of grammatical sequences would be greater than that of ungrammatical sequences.

Table 4 shows the mean COS for each predictable position of grammatical and ungrammatical sequences for two rules. For the inversion rule, credibility intervals showed that mean COS of the elements of grammatical sequences were substantially greater than those of ungrammatical sequences in position seven and nine for the trained networks. With regard to the performance of untrained networks, credibility intervals showed that mean COS 
of the elements of grammatical sequences were similar to those of ungrammatical sequences in all positions. That is, the trained networks predicted the correct tone type more strongly than the other tone type in the violating positions seven and nine, suggesting that the network was able to correctly predict the tones in the corresponding positions in the inversion rule after training. For the retrograde rule, mean COS of the elements of grammatical sequences were substantially greater than those of ungrammatical sequences only in position seven, but not position nine for the trained networks ${ }^{3}$. The performance of grammatical sequences was similar to that of ungrammatical sequences in all positions for the untrained networks. That is, the network has only a limited ability to predict the tones in the corresponding positions in the retrograde rule.

\section{Table 4}

Mean COS for each predictable position of grammatical and ungrammatical sequences for two rules.

\begin{tabular}{|c|c|c|c|c|c|c|c|c|c|c|c|c|c|}
\hline & \multirow{3}{*}{ Position } & \multicolumn{7}{|c|}{ Inversion } & \multicolumn{5}{|c|}{ Retrograde } \\
\hline & & \multicolumn{2}{|c|}{ G } & \multicolumn{2}{|c|}{ UG } & \multicolumn{2}{|c|}{$95 \% C I$} & \multicolumn{2}{|c|}{ G } & \multicolumn{2}{|c|}{ UG } & \multicolumn{2}{|c|}{$95 \% C I$} \\
\hline & & M & SE & M & $\mathrm{SE}$ & lower & upper & M & SE & M & SE & lower & upper \\
\hline \multirow{5}{*}{ trained } & Six & 0.87 & 0.02 & 0.87 & 0.02 & & $\mathrm{a}$ & 0.72 & 0.02 & 0.71 & 0.02 & $-0.00^{\mathrm{b}}$ & 0.01 \\
\hline & Seven & 0.86 & 0.02 & 0.60 & 0.02 & 0.18 & 0.35 & 0.85 & 0.02 & 0.66 & 0.02 & 0.14 & 0.24 \\
\hline & Eight & 0.74 & 0.03 & 0.74 & 0.03 & -0.01 & 0.01 & 0.56 & 0.02 & 0.56 & 0.02 & -0.01 & 0.01 \\
\hline & Nine & 0.81 & 0.02 & 0.56 & 0.02 & 0.16 & 0.33 & 0.75 & 0.01 & 0.75 & 0.02 & -0.04 & 0.05 \\
\hline & Ten & 0.86 & 0.02 & 0.85 & 0.02 & $-0.00^{b}$ & 0.03 & 0.69 & 0.02 & 0.67 & 0.02 & $-0.00^{\mathrm{b}}$ & 0.03 \\
\hline
\end{tabular}

\footnotetext{
${ }^{3}$ Li \& Liu (2018), using 100 instead of the current 10 epochs, but otherwise identical simulations, found that the mean COS of the elements of grammatical sequences were substantially greater than those of ungrammatical sequences in both position seven and nine.
} 


\begin{tabular}{cccccccccccccc} 
& Six & 0.65 & 0.02 & 0.65 & 0.02 & & a & 0.67 & 0.02 & 0.67 & 0.02 & $-0.00^{\mathrm{b}}$ & $0.00^{\mathrm{c}}$ \\
& Seven & 0.66 & 0.02 & 0.64 & 0.02 & -0.02 & 0.06 & 0.68 & 0.02 & 0.69 & 0.02 & -0.04 & 0.01 \\
untrained & Eight & 0.66 & 0.02 & 0.66 & 0.02 & -0.01 & 0.01 & 0.67 & 0.02 & 0.67 & 0.02 & -0.01 & $0.00^{\mathrm{c}}$ \\
& Nine & 0.66 & 0.02 & 0.67 & 0.02 & -0.05 & 0.04 & 0.67 & 0.02 & 0.68 & 0.02 & -0.05 & 0.02 \\
& Ten & 0.67 & 0.02 & 0.67 & 0.02 & $-0.00^{\mathrm{b}}$ & 0.01 & 0.66 & 0.02 & 0.65 & 0.02 & -0.01 & 0.02 \\
\hline
\end{tabular}

Note. $\mathrm{G}=$ grammatical, $\mathrm{UG}=$ ungrammatical. ${ }^{\mathrm{a}}$ No valid t-test was conducted because the standard error of difference is $0 .{ }^{\mathrm{b}}$ lower bound $\in(-0.005,0) .{ }^{\mathrm{c}}$ upper bound $\in(0,0.005)$.

\subsubsection{Characteristic behavior of the networks}

We evaluate how the typical performance of the networks compares to human data. As for study 1 , the effects of learning were calculated for each network by subtracting the z-scores of the untrained networks from the z-scores of the trained networks. Similarly, human participants' learning was assessed by subtracting the mean z-scores of the control group from that of the experimental group. The differences in z-scores on the inversion and retrograde were then plotted (see Fig. 6). The area covered by the human participants (mean \pm $1 S E)$ is also plotted in Fig. 6.

With regards to the human performance, the graph showed overlap in performance between the SRN and human's discrimination performance. Specifically, a substantial number of the SRNs fell within the area covered by the human data (28/150 for inversion, $43 / 150$ for retrograde, see Fig. 6), suggesting that the characteristic performance of the SRN matches reasonably well people's sensitivity to different non-local structures. 


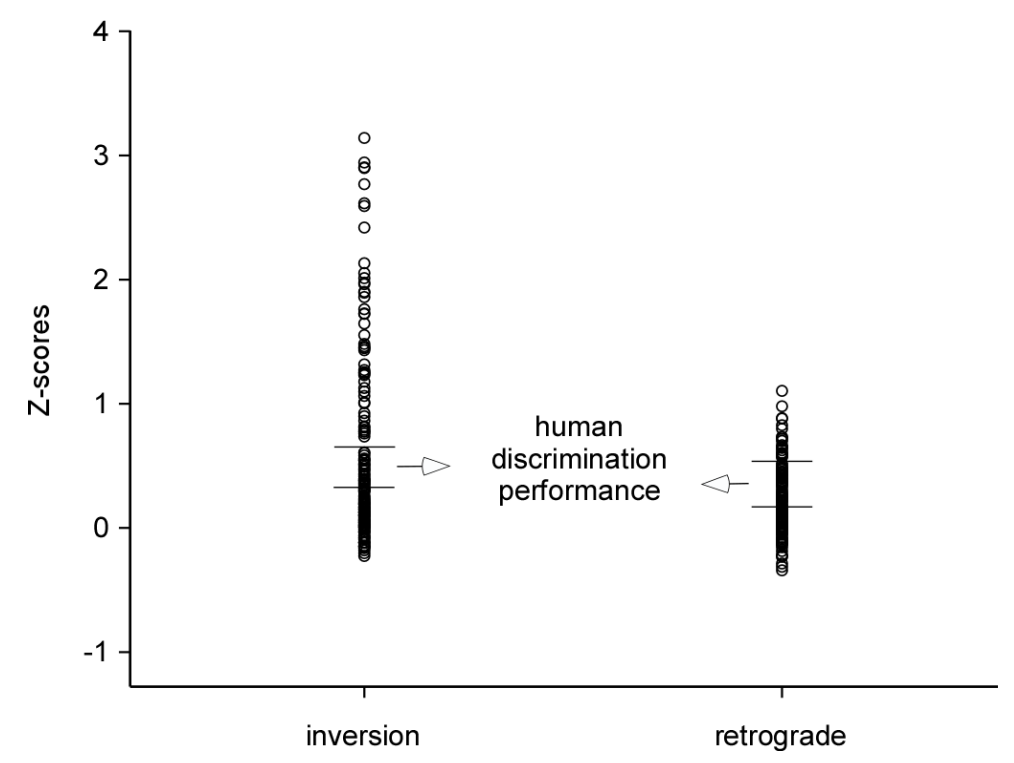

Fig. 6. Scatter plot shows the difference between the trained and untrained networks' z-scores on the retrograde and the $\mathrm{z}$-scores on the inversion. The area covered by the human participants (mean $\pm 1 S E$ ) is also plotted.

\section{General discussion}

The aim of the research presented here was to investigate the importance of prior knowledge and the nature of memory buffer in implicit learning of tonal symmetries. In study 1, we found that the SRN showed considerably better performance when a ping-ze coding (like being artificially provided prior knowledge of ping-ze categories) rather than a tone coding was used. These results enabled clear predictions for human participants, that is, learning the inversion rule without prior knowledge on how to divide the four terminals into the two categories may be very difficult, which was tested in study 2 . The results of Study 2 showed that participants did not learn the inversion symmetry based on an arbitrary classification of tones (i.e. in the absence of prior knowledge of the actually used ping-ze categories), confirming the predictions of the model. Learning has been found over ping ze 
categories for inversions in Jiang et al (2012; 8\% above control), in Li et al (2013; 7\% above control) and Qiao et al (2018; 6\% above control with different stimuli). Jointly, the results of study 1 and 2 indicated the importance of prior knowledge in implicit learning of symmetrical structures beyond finite state. The problem for people, unlike the SRN, may have been inconsistent prior knowledge, rather than no prior knowledge (as Chinese are taught in school a particular way of classifying ping and ze tones). Thus, the model predicts that non-Chinese who have learnt to identify the tones, but not the ping ze classification, should struggle with learning the inversion; Ling et al. (2018) present evidence consistent with this prediction using Western participants, but also a cross cultural difference in learning global rules versus chunks which complicates that comparison. Furthermore, results of study 3 showed that inversions were learnt more easily than retrogrades by the SRN, as Li et al. (2013) had shown for people, suggesting that functionally the memory buffer used in implicit learning is similar to the SRN's, i.e. functionally more like a first in-first out buffer than a last in-first out buffer.

Jiang et al. (2012) provided evidence that participants could go beyond learning chunks and repetition patterns and implicitly learn to detect tonal inversions. The present studies established further that the learning of tonal inversions by an SRN was facilitated when the prior knowledge was artificially provided for the SRN, but constrained when prior knowledge of ping-ze categories was absent, consistent with people's behaviors. Consistent with the proposal of prior knowledge constraints in what structures can be implicitly learnt, Leung and Williams (2011) and subsequently, Chen et al. (2011) found people did not learn about a form-meaning regularity when a linguistically arbitrary variable was used; Ziori and Dienes (2008) showed prior beliefs influenced what was learnt in implicit concept formation; Kuhn 
and Dienes (2005) showed implicit learning of an inversion in music not apparently learned in letter strings (Shanks, Johnstone, \& Staggs, 1997) but used in music composition.

Nevertheless, existing computational models in the implicit learning literature rarely include possible effects of prior knowledge (e.g., Boucher \& Dienes, 2003; Cleeremans, 1993a; Servan-Schreiber \& Anderson, 1990; but see Altmann, 2002, who found network without assigned pre-training weights is unlikely to accurately model real people). Therefore, from the modeling point of view, we provided new evidence for the important contribution of prior knowledge to implicit learning. The principle can be generalized as that of using informed priors over parameter space in computational models, as urged by Lee and Vanpaemel (2018). We used prior knowledge in a mild way in choosing the limits of the parameter values to be explored, that is as based on prior simulations in implicit learning (Cleeremans \& Dienes, 2008). However, these limits were broad, and we in effect put a uniform over that space in characterizing the typical behavior of the SRN. The difference in learnability of the different problems was sufficiently clear even with a uniform.

We found the SRN could learn inversion symmetry generated by higher than finite-state grammars, thus demonstrating that symmetries do require a memory buffer to process, which was in line with past results (Kuhn \& Dienes, 2008). Crucially, we showed that inversions were learnt more easily than retrogrades by the SRN, suggesting that functionally the memory buffer used in implicit learning is more like a first in-first out buffer than a last in-first out buffer. The actual buffer used need not be strictly either first in-first out or last in-first out; and the buffer in SRN is computationally flexible with its exact properties constructed according to the error landscape it is trained in. Nonetheless, a given memory system may function more 
like a first in-first out or last in-first out buffer, or find it easier to be trained to act like one of those rather than the other. In this sense, our results suggest that functionally the memory buffer used in implicit learning is more like a first in-first out buffer than a last in-first out buffer, which is consistent with previous human studies (Li et al., 2013; Uddén et al., 2012). Future research is needed to determine whether the SRN-type buffer really matches the computational properties of the buffer used in implicit learning (cf. Cleeremans, 1993b; Jones \& Mclaren, 2009; see also French, Addyman, \& Mareschal, 2011, for why the SRN-type buffer needs to be changed in some learning contexts).

The inversion and retrograde rules in the present research take the form of what Marcus (2001) calls "operations over variables". That is, an inversion or a retrograde is an operation that applies to a vector, and can in principle apply to a vector of any length (Dienes \& Longuet-Higgins, 2004). However, we used strings of fixed length, so the SRN (and the people in Jiang et al. (2012) and Li et al. (2013)) may not have learnt the symmetries per se. For example, for the inversion, the SRN may have learnt an association between corresponding positions in successive lines (see also Kuhn \& Dienes, 2008). For the retrograde, the SRN may have just learned to associate a specific position in one line (e.g. the $4^{\text {th }}$ ) with a specific position in the next line (e.g. the $\left.7^{\text {th }}\right)$. Thus, such long distance associations could explain the advantage of learning inversions over retrogrades, because in inversions, but not retrogrades, the associations occur at the same fixed distance for all positions (and retrogrades (corresponding to centre embeddings in nature language) have been argued to be especially difficult because of memory cost in syntactic prediction locality theory, Gibson, 1998). The analyses of non-local dependency learning by SRN in the present study provided 
indications for this explanation: the trained SRN network was able to correctly predict the tones in both positions seven and nine for the inversion rule; while for the retrograde rule, the trained SRN network could only correctly predict the tones in position seven, but not position nine with a longer distance before the prediction (relative to the distance of associations in reversion rule) was satisfied and thus requiring enhanced memory cost for maintaining that prediction. In order to show people (and the SRN) have learnt the symmetries per se, further research needs to show whether they can generalize their knowledge to different lengths of stimuli.

To conclude, by artificially providing prior knowledge of ping-ze categories for the SRN, the present study found the models showed better performance; while by using an arbitrary classification of tones, we showed that people couldn't learn a tonal inversion, which demonstrating the important role prior knowledge played in implicit learning of tonal symmetries. Furthermore, SRN simulation also showed that inversions were learnt more easily than retrogrades, suggesting that functionally the memory buffer used in implicit learning is more like a first in-first out buffer than a last in-first out buffer. 


\section{Conflict of interest}

The authors declare that the present study was conducted without any commercial or financial relationships that could be construed as a potential conflict of interest.

\section{Acknowledgements}

This work was supported by the National Natural Science Foundation of China (Grant no. 32071051, 31800912 and 32000770), Major project of medicine science and technology of

PLA (Grant no. AWS17J012) and ESRC (Grant no. ES/P009522/1). 


\section{References}

Altmann, G. T. M. (2002). Learning and development in neural networks - the importance of prior experience. Cognition, 85, B43-B50.

Altmann, G. T. M., \& Dienes, Z. (1999). Rule learning by seven-month-old infants and neural networks. Science, 284, 875a.

Baguley, T. (2012). Serious stats: A guide to advanced statistics for the behavioral sciences. Basingstoke, England: Palgrave Macmillan.

Balch, W. R. (1981). The role of symmetry in the good continuation ratings of two-part tonal melodies. Perception \& Psychophysics. 29(1), 47-55.

Boucher, L., \& Dienes, Z. (2003). Two ways of learning associations. Cognitive Science, 27, $807-842$.

Brooks, L. R., \& Vokey, J. R. (1991). Abstract analogies and abstracted grammars: Comments on Reber (1989) and Mathews et al. (1989). Journal of Experimental Psychology: General, 120(3), 316-323.

Caldwell-Harris, C., Ladd, R., Dediu, D., Biller, A. \& Christiansen, M.H. (2015). Factors influencing sensitivity to lexical tone in an artificial language: Implications for L2 learning. Studies in Second Language Acquisition, 37, 335-357.

Chen, W., Guo, X., Tang, J., Zhu, L., Yang, Z., \& Dienes, Z. (2011). Unconscious structural knowledge of form-meaning connections. Consciousness and Cognition, 20, $1751-1760$.

Chomsky, N. (1959). On certain formal properties of grammars. Information and Control, 2 , $137-167$. 
Christiansen, M. H., \& Chater, N. (1999). Toward a connectionist model of recursion in human linguistic performance. Cognitive Science, 23(2), 157-205.

Christiansen, M.H., Dale, R. \& Reali, F. (2010). Connectionist explorations of multiple-cue integration in syntax acquisition. In S.P. Johnson (Ed.), Neoconstructivism: The new science of cognitive development (pp. 87-108). New York, NY: Oxford University Press.

Cleeremans, A. (1993a). Attention and awareness in sequence learning. In Proceedings of the 15th Annual Conference of the Cognitive Science Society. Hillsdale, NJ: Lawrence Erlbaum Associates.

Cleeremans, A. (1993b). Mechanisms of implicit learning: Connectionist models of sequence processing. Cambridge, MA: MIT Press.

Cleeremans, A., Destrebecqz, A., \& Boyer, M. (1998). Implicit learning: News from the front. Trends in cognitive sciences, 2(10), 406-416.

Cleeremans, A., \& Dienes, Z. (2008). Computational models of implicit learning. In R. Sun (Ed.), Cambridge Handbook of Computational Psychology (pp 396-421). London, UK: Cambridge University Press.

De Vries, M., Monaghan, P., Knecht, S., \& Zwitserlood, P. (2008) Syntactic structure and artificial grammar learning: The learnability of embedded hierarchical structures. Cognition, 107, 763-774.

Dienes, Z. (2012). Conscious versus unconscious learning of structure. In P. Rebuschat \& J. Williams (Eds.), Statistical learning and language acquisition (pp. 337-364). Berlin, Germany: Mouton de Gruyter Publishers. 
Dienes, Z. (2014). Using Bayes to get the most out of non-significant results. Frontiers in Psychology, 5: 781.

Dienes, Z. (in press). How to use and report Bayesian hypothesis tests. Psychology of Consciousness: Theory, Research, and Practice.

Dienes, Z., Altmann, G. T. M., \& Gao, S. J. (1999). Mapping across domains without feedback: A neural network model of transfer of implicit knowledge. Cognitive Science, $23,53-82$.

Dienes, Z., \& Longuet-Higgins, C. (2004). Can musical transformations be implicitly learnt? Cognitive Science, 28, 531-558.

Dienes, Z., \& Scott, R. (2005). Measuring unconscious knowledge: Distinguishing structural knowledge and judgment knowledge. Psychological Research, 69, 338-351.

Dulany, D. E., Carlson, R. A., \& Dewey, G. I. (1984). A case of syntactical learning and judgment: How conscious and how abstract. Journal of Experimental Psychology: General, 113(4), 541-555.

Elman, J. L. (1990). Finding structure in time. Cognitive science, 14(2), 179-211.

Fitch, W. T., \& Friederici, A. D. (2012). Artificial grammar learning meets formal language theory: an overview. Philosophical Transactions of the Royal Society B: Biological Sciences, 367, 1933-1955.

French, R. M., Addyman, C., \& Mareschal, D. (2011). TRACX: A recognition-based connectionist framework for sequence segmentation and chunk extraction. Psychological review, 118(4), 614-636.

Gazdar, G., Klein, E., Pullum, G. K., \& Sag, I. A. (1985). Generalized phrase structure 
grammar. Cambridge, MA: Harvard University Press.

Gibson, E. (1998). Linguistic complexity: Locality of syntactic dependencies. Cognition, 68, $1-76$.

Jamieson, R. K., \& Mewhort, D. J. K. (2009). Applying an exemplar model to the artificial-grammar task: Inferring grammaticality from similarity. The Quarterly Journal of Experimental Psychology, 62(3), 550-575.

Janacsek, K., \& Nemeth, D. (2015). The puzzle is complicated: When should working memory be related to implicit sequence learning, and when should it not? Cortex, 64, $411-412$.

Jeffreys, H. (1939/1961). The Theory of Probability, 1st/3rd Edn. Oxford, England: Oxford University Press.

Jiang, S., Zhu, L., Guo, X., Ma, W., Yang, Z., \& Dienes, Z. (2012). Unconscious structural knowledge of tonal symmetry: Tang poetry redefines limits of implicit learning. Consciousness and cognition, 21, 476-486.

Jones, F. W., \& McLaren, I. P. L. (2009). Human sequence learning under incidental and intentional conditions. Journal of Experimental Psychology: Animal Behavior Processes, 35(4), 538-553.

Joshi, A. K., Vijay-Shanker, K., \& Weir, D. J. (1991). The convergence of mildly context-sensitive formalisms. In P. Sells, S. M. Shieber, \& T. Wasow (Eds.), Processing of linguistic structure (pp. 31-81). Cambridge, MA: MIT Press.

Jurchiş, R., Costea, A., Dienes, Z., Miclea, M., \& Opre, A. (in press). Evaluative conditioning of artificial grammars: Evidence that subjectively-unconscious structures 
bias affective evaluations of novel stimuli. Journal of Experimental Psychology: General.

Jurchiş, R., \& Opre, A. (2016). Unconscious Learning of Cognitive Structures with Emotional Components: Implications for Cognitive Behavior Psychotherapies. Cognitive Therapy and Research, 40, 230-244.

Kinder, A. (2000). The knowledge acquired during artificial grammar learning: Testing the predictions of two connectionist models. Psychological Research, 63(2), 95-105.

Kuhn, G., \& Dienes, Z. (2005). Implicit learning of non-local musical rules: Implicitly learning more than chunks. Journal of Experimental Psychology: Learning, Memory, and Cognition, 31(6), 1417-1432.

Kuhn, G., \& Dienes, Z. (2006). Differences in the types of musical regularity learnt in incidental-and intentional-learning conditions. The Quarterly Journal of Experimental Psychology. 59(10), 1725-1744.

Kuhn, G., \& Dienes, Z. (2008). Learning non-local dependencies. Cognition, 106, 184-206.

Lai, J. \& Poletiek, F. H. (2013). How "small” is "starting small" for learning hierarchical centre-embedded structures? Journal of Cognitive Psychology, 25 (4), 423-435.

Lee, M., \& Vanpaemel, W. (2018). Determining informative priors for cognitive models. Psychonomic Bulletin \& Review, 25, 114-127.

Leung, J., \& Williams, J. (2011). Constrains on implicit learning of form-meaning connections. Language Learning, 62(2), 634-662.

Lewicki, P. (1986). Processing information about covariations that cannot be articulated. Journal of Experimental Psychology: Learning, Memory, and Cognition, 12(1), 
$135-146$.

Li, F., Jiang, S., Guo, X., Yang, Z., \& Dienes, Z. (2013). The nature of the memory buffer in implicit learning: Learning Chinese tonal symmetries. Consciousness \& Cognition, 22 (3), 920-930.

Li, F., \& Liu, B. (2018). Which memory buffer does the implicit learning mechanism of nonlocal dependencies use: Evidence from neural network simulations. Journal of Psychological Science, 41(4), 796-802.

Ling, X., Li, F., Qiao, F., Guo, X., \& Dienes, Z. (2016). Fluency Expresses Implicit Knowledge of Tonal Symmetry. Frontiers in Psycholology, 7, 57.

Marcus, G. (2001). The algebraic mind. Cambridge, MIT: MIT Press.

Mareschal, D., \& French, R. M. (2017). Tracx2 : a connectionist autoencoder using graded chunks to model infant visual statistical learning. Philosophical Transactions of the Royal Society of London, 372, 20160057.

Martins, M. D., Gingras, B., Puig-Waldmueller, E., \& Fitch, W. T. (2017). Cognitive representation of "musical fractals": Processing hierarchy and recursion in the auditory domain. Cognition, 161, 31-45.

Martins, M. D., Martins, I. P., \& Fitch, W. T. (2016). A novel approach to investigate recursion and iteration in visual hierarchical processing. Behavior research methods 48 (4), $1421-1442$.

Misyak, J. B., Christiansen, M. H., \& Tomblin, J. B. (2009). Statistical learning of nonadjacencies predicts on-line processing of long-distance dependencies in natural language. In Proceedings of 31st Annual Cognitive Science Society Conference (pp. 
177-182). Austin, TX: Cognitive Science Society.

Neil, G. J., \& Higham, P. A. (2012). Implicit learning of conjunctive rule sets: An alternative to artificial grammars. Consciousness and Cognition, 21, 1393-1400.

Nguyen, D., \& Widrow, B. (1990). Improving the learning speed of 2-layer neural network by choosing initial values of the adaptive weights. Paper presented at the IEEE Proc. 1st Int. Joint Conf. Neural Networks.

Norman, E., \& Price, M. C. (2012). Social intuition as a form of implicit learning. Sequences of body movements are learned less explicitly than letter sequences. Advances in Cognitive Psychology, 8(2), 121-131.

Onnis, L., Destrebecqz, A., Christiansen, M.H., Chater, N. \& Cleeremans, A. (2015). Implicit learning of non-adjacent dependencies: A graded, associative account. In P. Rebuschat (Ed.), Implicit and explicit learning of languages (pp. 213-245). Amsterdam, Netherlands: John Benjamins.

Paciorek, A., \& Williams, J. N. (2015). Semantic Generalization in Implicit Language Learning. Journal of Experimental Psychology: Learning Memory and Cognition, 41, 989-1002.

Perruchet, P., \& Pacteau, C. (1990). Synthetic grammar learning - implicit rule abstraction or explicit fragmentary knowledge. Journal of Experimental Psychology: General, 119(3), $264-275$.

Perruchet, P., \& Rey, A. (2005) Does the mastery of hierarchical structures distinguishes humans from non-human primates? Psychonomic Bulletin \& Review, 12(2): 307-313.

Perruchet, P., \& Vinter, A. (1998). PARSER: A model for word segmentation. Journal of 
Memory and Language, 39, 246-263.

Poletiek, F. H. (2011). What in the world makes recursion so easy to learn? A statistical account of the staged input effect on learning a centre embedded hierarchical structure in AGL. Biolinguistics, 5 (1-2), 36-42.

Qiao, F., Sun, F., Li, F., Ling, X., Zheng, L., Li, L., Guo, X., \& Dienes, Z. (2018). Tonal symmetry induces fluency and sense of well-formedness. Frontiers in Psychology, 9, 165.

Reber, P.J., Batterink, L.J., Thompson, K.R. \& Reuveni, B. (2019). Implicit Learning: History and Applications. In A. Cleeremans (Ed.), Implicit Learning. Hove, UK: Psychology Press. Available here: http://reberlab.psych.northwestern.edu/wp-content/uploads/2012/07/Reber-Implicit-Le arning-2017.pdf

Rebuschat, P. (Ed.) (2015). Implicit and Explicit Learning of Languages. Amsterdam, Netherlands: John Benjamins.

Remillard, G. (2008). Implicit learning of second-, third-, and fourth-order adjacent and nonadjacent sequential dependencies. The Quarterly Journal of Experimental Psychology, 61, 400-424.

Rodriguez, P., Wiles, J., \& Elman, J. (1999). A Recurrent Neural Network that Learns to Count. Connection Science, 11(1), 5-40.

Rogers, J., Révész, A., \& Rebuschat, P. (2016). Implicit and explicit knowledge of inflectional morphology. Applied Psycholinguistics, 37, 781-81.

Rohrmeier, M., Dienes, Z., Guo, X., \& Fu, Q. (2014). Implicit learning and recursion. In F. 
Lowenthal \& L. Lefebvre (Eds), Language and Recursion (pp 67-85). New York, NY: Springer Verlag.

Rohrmeier, M., Fu, Q., \& Dienes, Z. (2012). Implicit learning of recursive, hierarchical grammatical structures. PLoS ONE, 7(10), e45885.

Rohrmeier, M., \& Widdess, R. (2017). Incidental learning of melodic structure of north indian music. Cognitive science, 41 (5), 1299-1327

Saffran, J. R., Newport, E. L., Aslin, R. N., Tunick, R. A., \& Barrueco, S. (1997). Incidental language learning: Listening (and learning) out of the corner of your ear. Psychological Science, 8(2), 101-105.

Servan-Schreiber, D., Cleeremans, A., \& McClelland, J. L. (1991). Graded state machines: The representation of temporal contingencies in simple recurrent networks. Machine Learning, 7, 161-193.

Servan-Schreiber, E., \& Anderson, J. R. (1990). Learning artificial grammars with competitive chunking. Journal of Experimental Psychology: Learning, Memory, and Cognition, 16(4), 592-608.

Shanks, D. R., Johnstone, T., \& Staggs, L. (1997). Abstraction processes in artificial grammar learning. The Quarterly Journal of Experimental Psychology Section A: Human Experimental Psychology, 50(1), 216-252.

Steedman, M. J. (2000). The syntactic process. Cambridge, MA: MIT Press.

Tanaka, K., \& Watanabe, K. (2013). Implicit transfer of reversed temporal structure in visuomotor sequence learning. Cognitive Science, 38, 565-579.

Tanaka, K., \& Watanabe, K. (2014). Implicit transfer of spatial structure in visuomotor 
sequence learning. Acta Psychologica, 153, 1-12.

Tillmann, B., Bharucha, J. J., \& Bigand, E. (2000). Implicit Learning of tonality: A self-organizing approach. Psychological Review, 107(4), 885-913.

Tunney, R. J., \& Altmann, G. T. M. (2001). Two modes of transfer in artificial grammar learning. Journal of Experimental Psychology: Learning, Memory, and Cognition, 27(3), 614-639.

Uddén, J., Ingvar, M., Hagoort, P., \& Petersson, K. M. (2012). Implicit acquisition of grammars with crossed and nested non-adjacent dependencies: Investigating the push-down stack model. Cognitive science, 36, 1078-1101.

Uddén, J., Ingvar, M., Hagoort, P., \& Petersson, K. M. (2017). Broca’s region: A causal role in implicit processing of grammars with crossed non-adjacent dependencies. Cognition, $164,188-198$.

Waroquier, L., Abadie, M.., \& Dienes, Z. (in press). Distinguishing the role of conscious and unconscious knowledge in Evaluative Conditioning. Cognition.

Zhang, Q., Li, L., Guo, X., Zheng, L., \& Zhou, C. (2020). Implicit learning of symmetry of human movement and gray matter density: evidence against pure domain general and pure domain specific theories of implicit learning. International Journal of Psychophysiology, 147, 60-71.

Ziori, E., \& Dienes, Z. (2006). Subjective measures of unconscious knowledge of concepts. Mind \& Society, 5(1), $105-122$.

Ziori, E., \& Dienes, Z. (2008). How does prior knowledge affect implicit and explicit concept learning? The Quarterly Journal of Experimental Psychology, 61(4), 601-624. 
Ziori, E., Pothos, E., \& Dienes, Z. (2014). Role of Prior Knowledge in Implicit and Explicit Learning of Artificial Grammars. Consciousness and Cognition, 28(1), 1-16. 